Claremont Colleges

Scholarship@ Claremont

All HMC Faculty Publications and Research

HMC Faculty Scholarship

$1-1-2016$

\title{
Existence of positive solutions for a semipositone p-Laplacian problem
}

Alfonso Castro

Harvey Mudd College

Djairo G. de Figueredo

Universidade Estadual de Campinas, Brazil

Emer Lopera

Universidad Nacional de Colombia, Colombia

\section{Recommended Citation}

(With D.G. de Figueiredo and E. Lopera) "Existence of positive solutions for a semipositone p-Laplacian problem", Proc. Roy. Soc. Edinburgh Sect. A 146 (2016), no. 3, 475-482.

This Article is brought to you for free and open access by the HMC Faculty Scholarship at Scholarship @ Claremont. It has been accepted for inclusion in All HMC Faculty Publications and Research by an authorized administrator of Scholarship @ Claremont. For more information, please contact scholarship@cuc.claremont.edu. 


\title{
Existence of positive solutions for a semipositone $p$-Laplacian problem
}

\author{
Alfonso Castro \\ Department of Mathematics, Harvey Mudd College, Claremont, \\ CA 91711, USA (castro@g.hmc.edu) \\ Djairo G. de Figueredo \\ Instituto de Matemática, Estatística e Computação Cientifica, \\ Universidade Estadual de Campinas, Caixa Postal 6065, Campinas, \\ SP 13083-859, Brazil (djairo@ime.unicamp.br)
}

\section{Emer Lopera}

Escuela de Matemáticas, Universidad Nacional de Colombia, Sede Medellín, Apartado Aéreo 3840, Medellín, Colombia

(edlopera@unal.edu.co)

(MS received 4 November 2014; accepted 25 March 2015)

\footnotetext{
We prove the existence of positive solutions to a semipositone $p$-Laplacian problem combining mountain pass arguments, comparison principles, regularity principles and a priori estimates.

Keywords: mountain pass theorem; semipositone problem; positive solutions; p-Laplacian; maximum principles; a priori estimates

2010 Mathematics subject classification: Primary 35J92; 35J20; 35J60
}

\section{Introduction}

In this paper we study the existence of positive weak solutions to the problem

$$
\left.\begin{array}{clrl}
-\Delta_{p} u & =\lambda f(u) & & \text { in } \Omega \\
u & =0 & & \text { on } \partial \Omega,
\end{array}\right\}
$$

where $\Delta_{p}(u)=\operatorname{div}\left(|\nabla u|^{p-2} \nabla u\right)$ denotes the $p$-Laplacian operator, $p>2 . \Omega$ is an open smooth bounded domain in $\mathbb{R}^{N}, N>2$. The function $f: \mathbb{R} \rightarrow \mathbb{R}$ is a differentiable function with $f(0)<0$ (semipositone). We assume that there exist $q \in(p-1, N p /(N-p)-1), A>0, B>0$ such that

$$
\left.\begin{array}{cl}
A\left(u^{q}-1\right) \leqslant f(u) \leqslant B\left(u^{q}+1\right) & \text { for } u>0 \\
f(u)=0 & \text { for } u \leqslant-1 .
\end{array}\right\}
$$


We also assume an Ambrosetti-Rabinowitz type of condition, namely that there exist $\theta>p$ and $M \in \mathbb{R}$ such that

$$
u f(u) \geqslant \theta F(u)+M
$$

where

$$
F(u)=\int_{0}^{u} f(s) \mathrm{d} s .
$$

The assumption $f(0)<0$ implies that $u=0$ is not a subsolution to (1.1), making the finding of positive solutions rather challenging; this was pointed out in [6].

The aim of this paper is to prove the following result.

Theorem 1.1. There exists $\lambda^{*}>0$ such that if $\lambda \in\left(0, \lambda^{*}\right)$, then the problem (1.1) has a positive weak solution $u_{\lambda} \in C^{1, \beta}(\bar{\Omega})$ for some $\beta \in(0,1)$.

Our results extend [1, theorem 1.1], where the case $p=2$ was studied. Extending such a theorem to $p>2$ is not straightforward due to the lack of regularity and linearity of $\Delta_{p}$. Associated to (1.1) we have a functional, which will be defined in the next section. We show that this functional has a critical point of mountain pass type and, consequently, a weak solution of (1.1) for appropriate values of $\lambda>0$. Finally, using order properties of $-\Delta_{p}$, we prove that by further restricting $\lambda$ such a solution is actually positive. For recent results on semipositone problems the reader is referred to $[2,3]$.

\section{Preliminary results}

Let $W_{0}^{1, p}(\Omega)$ denote the Banach space of functions in $L^{p}(\Omega)$ with first-order partial derivatives in $L^{p}(\Omega)$ and vanishing on $\partial \Omega$. By a weak solution to (1.1) we mean an element $u \in W_{0}^{1, p}(\Omega)$ such that

$$
\int_{\Omega}|\nabla u|^{p-2}\langle\nabla u, \nabla \phi\rangle \mathrm{d} x=\lambda \int_{\Omega} f(u) \phi \mathrm{d} x
$$

for all $\phi \in W_{0}^{1, p}(\Omega)$. We denote by $\|\cdot\|_{s}$ the norm in the space $L^{s}(\Omega)$ and by $\|\cdot\|_{1 . p}$ the norm in the Sobolev space $W_{0}^{1, p}(\Omega)$.

Associated to (1.1) we have the functional $J_{\lambda}: W_{0}^{1, p}(\Omega) \rightarrow \mathbb{R}$ defined by

$$
J_{\lambda}(u):=\int_{\Omega} \frac{|\nabla u(x)|^{p}}{p} \mathrm{~d} x-\int_{\Omega} \lambda F(u(x)) \mathrm{d} x,
$$

where

$$
F(s):=\int_{0}^{s} f(r) \mathrm{d} r .
$$

It is well known that $J_{\lambda}$ is a functional of class $C^{1}$ (see [7]) and that the critical points of the functional $J_{\lambda}$ are the weak solutions of (1.1). The proof of theorem 1.1 consists of two main steps:

(i) the proof of existence of one solution via the mountain pass theorem,

(ii) the proof that for proper values of $\lambda$ the solution is indeed positive. 
It follows from (1.2) that there exist positive real numbers $A_{1}, B_{1}$ such that

$$
F(u) \leqslant B_{1}\left(|u|^{q+1}+1\right) \quad \text { for all } u \in \mathbb{R}
$$

and

$$
F(u) \geqslant A_{1}\left(|u|^{q+1}-1\right) \text { for all } u \geqslant 0 .
$$

For simplicity of the notation, we define $r=1 /(q+1-p)>0$. Let $\varphi \in W_{0}^{1, p}(\Omega)$ denote a positive differentiable function with $\|\varphi\|_{1, p}=1$. Let us define the constant

$$
c=\left(2 p^{-1} A_{1}^{-1}\|\varphi\|_{q+1}^{-q-1}\right)^{r},
$$

which will be used in the next lemma.

The next two lemmas prove that $J_{\lambda}$ satisfies the geometric hypotheses of the mountain pass theorem.

LEMma 2.1. There exists $\lambda_{1}>0$ such that if $\lambda \in\left(0, \lambda_{1}\right)$, then $J_{\lambda}\left(c \lambda^{-r} \varphi\right) \leqslant 0$.

Proof. Let $s=c \lambda^{-r}$, with $c$ and $r$ as defined above. Hence, due to (2.4),

$$
\begin{aligned}
J_{\lambda}(s \varphi) & =\int_{\Omega}\left\{\frac{|\nabla(s \varphi)|^{p}}{p}-\lambda F(s \varphi)\right\} \mathrm{d} x \\
& \leqslant \frac{s^{p}}{p}-\lambda A_{1} \int_{\Omega}\left(s^{q+1} \varphi^{q+1}-1\right) \mathrm{d} x \\
& =\frac{s^{p}}{p}-A_{1} s^{q+1}\|\varphi\|_{q+1}^{q+1} \lambda+\lambda A_{1}|\Omega| \\
& =c^{p}\left\{\frac{\lambda^{-r p}}{p}-\lambda A_{1} c^{q+1-p} \lambda^{-r(q+1)}\|\varphi\|_{q+1}^{q+1}\right\}+\lambda A_{1}|\Omega| .
\end{aligned}
$$

Substituting (2.5) into (2.6) yields

$$
\begin{aligned}
J_{\lambda}(s \varphi) & \leqslant c^{p}\left(\frac{\lambda^{-r p}}{p}-\frac{2}{p} \lambda^{1-r(q+1)}\right)+\lambda A_{1}|\Omega| \\
& =c^{p} \lambda^{-r p}\left(\frac{1}{p}-\frac{2}{p} \lambda^{1+r p-r(q+1)}\right)+\lambda A_{1}|\Omega| \\
& =-c^{p} \lambda^{-r p} \frac{1}{p}+\lambda A_{1}|\Omega| .
\end{aligned}
$$

Taking $\lambda_{1}<\min \left\{1,\left(p A_{1} c^{-p}|\Omega|\right)^{-1 /(1+p r)}\right\}$, the lemma is proven.

LEMMA 2.2. There exist $\tau>0, c_{1}>0$, and $\lambda_{2} \in(0,1)$ such that if $\|u\|_{1, p}=\tau \lambda^{-r}$, then $J_{\lambda}(u) \geqslant c_{1}\left(\tau \lambda^{-r}\right)^{p}$ for all $\lambda \in\left(0, \lambda_{2}\right)$.

Proof. By the Sobolev embedding theorem there exists $K_{1}>0$ such that if $u \in$ $W_{0}^{1, p}(\Omega)$, then $\|u\|_{q+1} \leqslant K_{1}\|u\|_{1, p}$. Let

$$
\tau=\min \left\{\left(2 p K_{1}^{q+1} B_{1}\right)^{-r}, c\|\varphi\|_{1, p}\right\} .
$$


If $\|u\|_{W_{0}^{1, p}}=\tau \lambda^{-r}$, then

$$
\begin{aligned}
J_{\lambda}(u) & =\frac{\left(\tau \lambda^{-r}\right)^{p}}{p}-\int_{\Omega} \lambda F(u) \\
& \geqslant \frac{\left(\tau \lambda^{-r}\right)^{p}}{p}-\lambda \int_{\Omega} B_{1}|u|^{q+1}-\lambda|\Omega| B_{1} \\
& \geqslant \frac{\left(\tau \lambda^{-r}\right)^{p}}{p}-\lambda B_{1} K_{1}^{q+1}\|\nabla u\|_{p}^{q+1}-\lambda|\Omega| B_{1} \\
& =\frac{\left(\tau \lambda^{-r}\right)^{p}}{p}-\lambda B_{1} K_{1}^{q+1}\left(\tau \lambda^{-r}\right)^{q+1}-\lambda|\Omega| B_{1} \\
& =\lambda^{-r p}\left[\frac{\tau^{p}}{2 p}-\lambda^{1+r p}|\Omega| B_{1}\right] \\
& \geqslant \lambda^{-r p} \frac{\tau^{p}}{4 p}
\end{aligned}
$$

where we have used that $\tau \leqslant\left(2 p K_{1}^{q+1} B_{1}\right)^{-r}$ (see (2.8)). Taking $c_{1}=\tau^{p} /(4 p)$ and $\lambda_{2}=\tau^{p /(1+r p)}\left(4 p B_{1}|\Omega|\right)^{-1 /(1+r p)}$, the lemma is proven.

Next, using the mountain pass theorem we prove that (1.1) has a solution $u_{\lambda} \in$ $W_{0}^{1, p}(\Omega)$.

Lemma 2.3. Let $\lambda_{3}=\min \left\{\lambda_{1}, \lambda_{2}\right\}$. There exists $c_{2}>0$ such that, for each $\lambda \in$ $\left(0, \lambda_{3}\right)$, the functional $J_{\lambda}$ has a critical point $u_{\lambda}$ of mountain pass type that satisfies $J_{\lambda}\left(u_{\lambda}\right) \leqslant c_{2} \lambda^{-p r}$.

Proof. First we show that $J_{\lambda}$ satisfies the Palais-Smale condition.

Assume that $\left\{u_{n}\right\}_{n}$ is a sequence in $W_{0}^{1, p}(\Omega)$ such that $\left\{J_{\lambda}\left(u_{n}\right)\right\}_{n}$ is bounded and $J_{\lambda}^{\prime}\left(u_{n}\right) \rightarrow 0$. Hence, there exists $\nu>0$ such that $\left\langle J_{\lambda}^{\prime}\left(u_{n}\right), u_{n}\right\rangle \leqslant\left\|\nabla u_{n}\right\|_{p}$ for $n \geqslant \nu$. Thus,

$$
-\left\|\nabla u_{n}\right\|_{p}^{p}-\left\|\nabla u_{n}\right\|_{p} \leqslant-\lambda \int_{\Omega} f\left(u_{n}\right) u_{n} \mathrm{~d} x \text { for } n \geqslant \nu .
$$

Let $K$ be a constant such that $\left|J_{\lambda}\left(u_{n}\right)\right| \leqslant K$ for all $n=1,2, \ldots$. From (1.3), we obtain

$$
\frac{1}{p}\left\|\nabla u_{n}\right\|_{p}^{p}-\frac{\lambda}{\theta} \int_{\Omega} f\left(u_{n}\right) u_{n} \mathrm{~d} x+\frac{\lambda}{\theta} M|\Omega| \leqslant \frac{1}{p}\left\|\nabla u_{n}\right\|_{p}^{p}-\lambda \int_{\Omega} F\left(u_{n}\right) \mathrm{d} x \leqslant K .
$$

From the last two inequalities we have

$$
\left(\frac{1}{p}-\frac{1}{\theta}\right)\left\|\nabla u_{n}\right\|_{p}^{p}-\frac{1}{\theta}\left\|\nabla u_{n}\right\|_{p} \leqslant K-\frac{\lambda}{\theta} M|\Omega| .
$$

This proves that $\left\{u_{n}\right\}$ is a bounded sequence. Thus, without loss of generality, we may assume that $\left\{u_{n}\right\}$ converges weakly. Let $u \in W_{0}^{1, p}(\Omega)$ be its weak limit. Since $q<N p /(N-p)$, by the Sobolev embedding theorem we may assume that $\left\{u_{n}\right\}$ converges to $u$ in $L^{q}(\Omega)$. These assumptions and Hölder's inequality imply

$$
\int_{\Omega} \lambda f\left(u_{n}\right)\left(u_{n}-u\right) \rightarrow 0
$$


From (2.10) and $\lim _{n \rightarrow+\infty} J_{\lambda}^{\prime}\left(u_{n}\right)=0$ we have

$$
\lim _{n \rightarrow+\infty} \int_{\Omega}\left|\nabla u_{n}\right|^{p-2} \nabla u_{n}\left(\nabla u_{n}-\nabla u\right) \mathrm{d} x=0 .
$$

Using again that $u$ is the weak limit of $\left\{u_{n}\right\}$ in $W_{0}^{1, p}(\Omega)$ we also have

$$
\lim _{n \rightarrow+\infty} \int_{\Omega}|\nabla u|^{p-2} \nabla u\left(\nabla u_{n}-\nabla u\right) \mathrm{d} x=0 .
$$

By Hölder's inequality,

$$
\begin{aligned}
\int_{\Omega}\left(\left|\nabla u_{n}\right|^{p-2} \nabla u_{n}-|\nabla u|^{p-2} \nabla u\right)\left(\nabla u_{n}-\nabla u\right) \mathrm{d} x \\
\quad \geqslant\left\|\nabla u_{n}\right\|_{p}^{p}-\|\nabla u\|_{p}\left\|\nabla u_{n}\right\|_{p}^{p-1}-\left\|\nabla u_{n}\right\|_{p}\|\nabla u\|_{p}^{p-1}+\|\nabla u\|_{p}^{p} \\
\quad=\left(\left\|\nabla u_{n}\right\|_{p}^{p-1}-\|\nabla u\|_{p}^{p-1}\right)\left(\left\|\nabla u_{n}\right\|_{p}-\|\nabla u\|_{p}\right) \\
\quad \geqslant 0 .
\end{aligned}
$$

From (2.11)-(2.13),

$$
\lim _{n \rightarrow \infty}\left(\left\|\nabla u_{n}\right\|_{p}^{p-1}-\|\nabla u\|_{p}^{p-1}\right)\left(\left\|\nabla u_{n}\right\|_{p}-\|\nabla u\|_{p}\right)=0,
$$

which implies that $\lim _{n \rightarrow \infty}\left\|\nabla u_{n}\right\|_{p}=\|\nabla u\|_{p}$. Since $u_{n} \rightarrow u, u_{n} \rightarrow u$ in $W_{0}^{1, p}$. This proves that $J_{\lambda}$ satisfies the Palais-Smale condition.

From (2.6) we see that

$$
\begin{aligned}
\max \left\{J_{\lambda}(s \varphi) ; s \geqslant 0\right\} & \leqslant \frac{C^{1+p r}\left((q+1)^{r(q-p)}-p\right)}{D^{p r} p(q+1)^{r(q+1)}} \lambda^{-p r}+\lambda A_{1}|\Omega| \\
& :=c_{2}^{\prime} \lambda^{-p r}+\lambda A_{1}|\Omega| \leqslant c_{2}^{\prime} \lambda^{-p r}+A_{1}|\Omega| \lambda^{-p r} \\
& :=c_{2} \lambda^{-p r},
\end{aligned}
$$

where $C=\|\nabla \varphi\|_{p}^{p}$ and $D=A_{1}\|\varphi\|_{q+1}^{q+1}$.

With this estimate and lemma 2.2 , the existence of $u_{\lambda} \in W_{0}^{1, p}(\Omega)$ such that $\nabla J_{\lambda}\left(u_{\lambda}\right)=0$ and

$$
c_{1}\left(\tau \lambda^{-r}\right)^{p} \leqslant J_{\lambda}\left(u_{\lambda}\right) \leqslant c_{2} \lambda^{-p r}
$$

follows by the mountain pass theorem.

REMARK 2.4. The solution $u_{\lambda} \in W_{0}^{1, p}(\Omega)$ is indeed in $C^{1, \alpha}(\bar{\Omega})$ (cf. [5]).

Lemma 2.5. Let $u_{\lambda}$ be as in lemma 2.3. Then there is a positive constant $M_{0}$ such that

$$
M_{0} \lambda^{-r} \leqslant\left\|u_{\lambda}\right\|_{\infty}
$$

Proof. We already know that there exists $c_{1}>0$ such that $J\left(u_{\lambda}\right) \geqslant c_{1} \lambda^{-r p}$. On the other hand, we have that $F(s) \geqslant \min F>-\infty$ and $f(s) s \leqslant B_{1}\left(|s|^{q+1}+|s|\right)$ for all 
$s \in \mathbb{R}$. Then there is a constant $C_{1}>0$ such that

$$
\begin{aligned}
\lambda \int_{\Omega} f\left(u_{\lambda}\right) u_{\lambda} \mathrm{d} x & =\int_{\Omega}\left|\nabla u_{\lambda}\right|^{p} \mathrm{~d} x \\
& =p J\left(u_{\lambda}\right)+p \lambda \int_{\Omega} F\left(u_{\lambda}\right) \mathrm{d} x \\
& \geqslant p C_{1} \lambda^{-r p}+p|\Omega| \lambda \min F \\
& \geqslant C_{1} \lambda^{-r p} .
\end{aligned}
$$

Thus, $\lim _{\lambda \rightarrow 0}\left\|u_{\lambda}\right\|_{\infty}=+\infty$. On the other hand, by (2.3),

$$
\begin{aligned}
\lambda \int_{\Omega} f\left(u_{\lambda}\right) u_{\lambda} \mathrm{d} x & \leqslant B_{1} \lambda \int_{\Omega}\left(\left|u_{\lambda}\right|^{q+1}+\left|u_{\lambda}\right|\right) \mathrm{d} x \\
& \leqslant B_{1} \lambda \int_{\Omega}\left(\left\|u_{\lambda}\right\|_{\infty}^{q+1}+\left\|u_{\lambda}\right\|_{\infty}\right) \mathrm{d} x \\
& \leqslant 2 B_{1}|\Omega| \lambda\left\|u_{\lambda}\right\|_{\infty}^{q+1},
\end{aligned}
$$

where we have used the fact that $0<\lambda<1$. Finally, taking $M_{0}=C_{1} / 2 B_{1}|\Omega|$, the lemma is proven.

Lemma 2.6. Let $u_{\lambda}$ be as in lemma 2.3. Then there exists $c_{3}>0$ such that

$$
\left\|u_{\lambda}\right\|_{1, p}^{p} \leqslant c_{3} \lambda^{-p r}
$$

for all $\lambda \in\left(0, \lambda_{3}\right)$.

Proof. By (1.3) and the definition of $u_{\lambda}$,

$$
\begin{aligned}
\lambda \int_{\Omega} \frac{\theta-p}{\theta} u_{\lambda} f\left(u_{\lambda}\right) \mathrm{d} x & \leqslant \lambda \int_{\Omega}\left(u_{\lambda} f\left(u_{\lambda}\right)-p F\left(u_{\lambda}\right)\right) \mathrm{d} x-\frac{\lambda p M|\Omega|}{\theta} \\
& =\int_{\Omega}\left(\left|\nabla u_{\lambda}\right|^{p}-p \lambda F\left(u_{\lambda}\right)\right) \mathrm{d} x-\frac{\lambda p M|\Omega|}{\theta} \\
& \leqslant c_{2} \lambda^{-r p}+\frac{\lambda p M|\Omega|}{\theta} \\
& \leqslant 2 c_{2} \lambda^{-r p}
\end{aligned}
$$

where we have used $0<\lambda<1$. Now the result follows from (2.18) and the fact that $u_{\lambda}$ is a weak solution of (1.1).

\section{Proof of theorem 1.1}

We prove theorem 1.1 by contradiction. Suppose there exists a sequence $\left\{\lambda_{j}\right\}_{j}, 1>$ $\lambda_{j}>0$ for all $j$, converging to 0 such that the measure $m\left(\left\{x \in \Omega ; u_{\lambda_{j}}(x) \leqslant 0\right\}\right)>0$. Letting $w_{j}=u_{\lambda_{j}} /\left\|u_{\lambda_{j}}\right\|_{\infty}$, we see that

$$
-\Delta_{p}\left(w_{j}\right)=\lambda_{j} f\left(u_{\lambda_{j}}\right)\left\|u_{\lambda_{j}}\right\|_{\infty}^{1-p} .
$$

From lemmas 2.5 and 2.6 there is a constant $C_{3}$ such that

$$
\left\|w_{j}\right\|_{1, p} \leqslant C_{3} .
$$


By [4, proposition 3.7] the sequence $w_{j}$ is uniformly bounded in $C^{1, \alpha}$ for some $\alpha \in(0,1)$. Hence, for any $\beta \in(0, \alpha)$, the sequence $w_{j}$ has a subsequence that converges in $C_{0}^{1, \beta}$. Let us denote its limit by $w$.

Next, using comparison principles, we prove that $w(x) \geqslant 0$.

Let $v_{0} \in W_{0}^{1, p}(\Omega)$ be the solution of

$$
\left.\begin{array}{rl}
-\Delta_{p} v_{0}=1 & \text { in } \Omega, \\
v_{0}=0 & \text { on } \partial \Omega .
\end{array}\right\}
$$

Let $K_{j}:=\lambda_{j} \min \{f(t) ; t \in \mathbb{R}\}\left\|u_{\lambda_{j}}\right\|_{\infty}^{1-p}$. Then the solution $v_{j}$ of the equation

$$
\left.\begin{array}{rlrl}
-\Delta_{p} v_{j} & =K_{j} & & \text { in } \Omega, \\
v & =0 & & \text { on } \partial \Omega
\end{array}\right\}
$$

is given by $v_{j}=\left(-K_{j}\right)^{1 /(p-1)} v_{0}$.

Since $\lambda_{j} f\left(u_{\lambda_{j}}\right)\left\|u_{\lambda_{j}}\right\|_{\infty}^{1-p} \geqslant K_{j}$, it follows by the comparison principle in [9] that $w_{j} \geqslant v_{j}$. Then the fact that $v_{j}(x) \rightarrow 0$ as $j \rightarrow 0$ implies that $w(x) \geqslant 0$ for all $x \in \Omega$.

Since, by hypothesis, $q>p-1$, we have $s=N p r /(N-p)>1$. This result, together with the Sobolev embedding theorem, (1.2) and lemma 2.6, gives

$$
\begin{aligned}
\int_{\Omega}\left|f\left(u_{\lambda_{j}}\right)\right|^{s}\left\|u_{\lambda_{j}}\right\|_{\infty}^{s(1-p)} \mathrm{d} x & \leqslant B^{s} 2^{s-1} \int_{\Omega}\left(\left|u_{\lambda_{j}}\right|^{(q+1-p) s}+1\right) \mathrm{d} x \\
& \leqslant C\left(\left\|u_{\lambda_{j}}\right\|_{1, p}^{N p /(N-p)}+1\right) \\
& \leqslant C\left(c_{3} \lambda_{j}^{-r N p /(N-p)}+1\right)
\end{aligned}
$$

where $C>0$ is a constant independent of $j$ and, without loss of generality, we have assumed $\left\|u_{\lambda_{j}}\right\|_{\infty} \geqslant 1$. From (3.5) and the fact that $r N p /(s N-s p)=1$ we see that $\left\{\lambda_{j} f\left(u_{\lambda_{j}}\right)\left\|u_{\lambda_{j}}\right\|_{\infty}^{1-p}\right\}$ is bounded in $L^{s}(\Omega)$, so we may assume that it converges weakly. Let $z \in L^{s}(\Omega)$ be the weak limit of such a sequence. Since $\left\|u_{\lambda_{j}}\right\|_{\infty}^{1-p} \lambda_{j} \rightarrow 0$ as $j \rightarrow+\infty$ and $f$ is bounded from below, $z \geqslant 0$. Now if $\phi \in C_{0}^{\infty}(\Omega)$, then

$$
\begin{aligned}
\int_{\Omega}\|\nabla w\|^{p-2}\langle\nabla w, \nabla \phi\rangle \mathrm{d} x & =\lim _{j \rightarrow \infty} \int_{\Omega}\left\|\nabla w_{j}\right\|^{p-2}\left\langle\nabla w_{j}, \nabla \phi\right\rangle \mathrm{d} x \\
& =\lim _{j \rightarrow \infty} \int_{\Omega}\left\|u_{\lambda_{j}}\right\|_{\infty}^{1-p}\left\|\nabla u_{\lambda_{j}}\right\|^{p-2}\left\langle\nabla u_{\lambda_{j}}, \nabla \phi\right\rangle \mathrm{d} x \\
& =\lim _{j \rightarrow \infty} \int_{\Omega}\left\|u_{\lambda_{j}}\right\|_{\infty}^{1-p} \lambda_{j} f\left(u_{\lambda_{j}}\right) \phi \mathrm{d} x \\
& =\int_{\Omega} z \phi \mathrm{d} x .
\end{aligned}
$$

Therefore, $-\Delta_{p} w=z$. Since $\left\|w_{j}\right\|_{\infty}=1, w \neq 0$. By Hopf's maximum principle for the $p$-Laplacian operator (see [8, theorem 5.1]), $w>0$ in $\Omega$ and

$$
\frac{\partial w}{\partial \nu}(x)<0 \quad \text { for all } x \in \partial \Omega .
$$

Here $\partial / \partial n$ denotes the outward unit normal derivative. Therefore, since $\left\{w_{j}\right\}_{j}$ converges in $C^{1, a}$ to $w$, for sufficiently large $j, w_{j}(x)>0$ for all $x \in \Omega$. Hence, 
$u_{\lambda_{j}}(x)>0$ for all $x \in \Omega$, which contradicts the assumption that

$$
m\left(\left\{x ; u_{\lambda_{j}}(x)<0\right\}\right)>0 .
$$

This contradiction proves theorem 1.1.

\section{Acknowledgements}

A.C. was partly supported by Grant no. 245966 from the Simons Foundation.

\section{References}

1 S. Caldwell, A. Castro, R. Shivaji and S. Unsurangsie. Positive solutions for classes of multiparameter elliptic semipositone problems. Electron. J. Diff. Eqns 2007 (2007), paper 96.

2 M. Chhetri and R. Shivaji. Existence of a positive solution for a $p$-Laplacian semipositone problem. Bound. Value Probl. 2005 (2005), 323-327.

3 M. Chhetri, and P. Girg. Existence of positive solutions for a class of superlinear semipositone systems. J. Math. Analysis Applic. 408 (2013), 781-788.

4 D. G. de Figueiredo, J. P. Gossez and P. Ubilla. Local superlinearity and sublinearity for the $p$-Laplacian. J. Funct. Analysis 257 (2009), 721-752.

$5 \quad$ M. Guedda and L. Veron. Quasilinear elliptic equations involving critical Sobolev exponents. Nonlin. Analysis 13 (1989), 879-902.

6 P. L. Lions. On the existence of positive solutions of semilinear elliptic equations. SIAM Rev. 24 (1982), 441-467.

$7 \quad$ P. H. Rabinowitz. Minimax methods in critical point theory with applications to differential equations. Regional Conference Series in Mathematics, vol. 65 (Providence, RI: American Mathematical Society, 1986).

8 P. Takáç. Degenerate elliptic equations in ordered Banach spaces and applications. In Nonlinear differential equations, Chapman and Hall/CRC Research Notes in Mathematics, vol. 404, pp. 111-196 (Boca Raton, FL: CRC Press, 1999).

9 P. Tolksdorf. On the Dirichlet problem for quasilinear equations in domains with conical boundary points. Commun. PDEs 8 (1983), 773-817. 